\title{
MESOSCALE ENGINEERING OF NANOCOMPOSITE NONLINEAR OPTICAL MATERIALS
}

\author{
R. F. Haglund, Jr., ${ }^{1}$ C. N. Afonso, ${ }^{2}$ L. C. Feldman, ${ }^{\prime}$ F. Gonella, ${ }^{3}$ G. Luepke, ${ }^{1}$ R. H. Magruder, \\ P. Mazzoldi, ${ }^{3}$ D. H. Osborne, ${ }^{1}$ J. Solis ${ }^{2}$ and R. A. Zuhr ${ }^{4}$ \\ 1 Vanderbilt University, Nashville TN 37235 \\ Instituta de Optica, CSIC, Madrid, Spain \\ 3 CNR-INFM, Universitá di Padova, Padova, Italy \\ ${ }^{4}$ Oak Ridge National Laboratory, Oak Ridge, TN 37831
}

\begin{abstract}
Complex nonlinear optical materials comprising elemental, compound or alloy quantum dots embedded in appropriate dielectric or semiconducting hosts may be suitable for deployment in photonic devices. Ion implantation, ion exchange followed by ion implantation, and pulsed laser deposition have all been used to synthesize these materials. However, the correlation between the parameters of energetic-beam synthesis and the nonlinear optical properties is still very rudimentary when one starts to ask what is happening at nanoscale dimensions. Systems integraion of complex nonlinear optical materials requires that the mesoscale materials science be well understood within the context of device structures. We discuss the effects of beam energy and energy density on quantum-dot size and spatial distribution, thermal conductivity, quantum-dot composition, crystallinity and defects - and, in turn, on the third-order optical susceptibility of the composite material. Examples from recent work in our laboratories are used to illustrate these effects.
\end{abstract}

\section{Introduction and Motivation}

Research groups around the world have reported the fabrication of metal quantum-dot composites (MQDCs) by such varied techniques as ion implantation,' ion exchange followed by ion implantation, ${ }^{2}$ sol-gel synthesis, ${ }^{3}$ sputtering ${ }^{4}$ and pulsed laser deposition. ${ }^{5}$ The motivation cited in these reports has been to synthesize materials suitable for all-optical switching, to take advantage of the short duration and high pulse repetition frequency of optical signals. Invariably cited in all these publications is the large third-order nonlinearity $\chi^{(3)}$ of these materials, which produces ai-

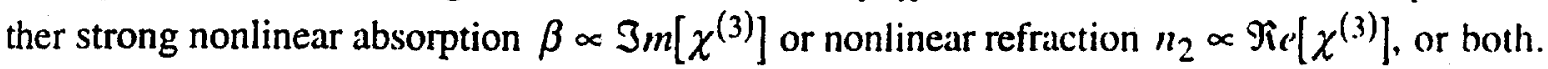

However, simply having a large value of $\chi^{(3)}$ is insufficient to justify deployment of these materials in all-optical switching technology. A much more complex set of problems is associated with optimizing the materials properties of MQDCs to provide an adequate nonlinear optical response within the constraints set by device functionality and operating conditions. In this context. the mesoscale properties of the MQDCs which affect high-frequency performance, such as thermal conductivity and defect properties, are of special concern.

Here we address a number of mesoscale materials properties which affect the size of the third-order nonlinearity, such as tunability, relaxation time, and high-frequency operation. Several beam-assisted processing techniques can produce either waveguide or layered structures; the challenge lies in adapting these techniques to produce self-assembling or patterned structures. Comparisons of the third-order nonlinear optical properties of MQDCs made by ion implantation, ion exchange followed by ion implantation, and pulsed laser deposition suggest that the greater regularity of layered structures, as found in pulsed laser deposition. leads to significant improvement. We also consider prospects for producing laterally structured or patterned nonlinear materials. 


\section{Physical and Optical Characteristics of Quantum-Dot Composites}

The arenas of concern for mesoscale engineering of a MQDC are illustrated in Figure 1 , and are circumscribed by the processes of optical excitation and relaxation which occur when the dot is irradiated by a short laser pulse. The physical and electronic structure of the quantum dot governs the third-order nonlinear response, particularly the quantumconfined component, and the dissipation of the excitation energy through electron-electron and electron-phonon interactions. The interface properties determine the transfer of thermal energy from the dot to the surrounding matrix, and are especially critical for relaxation rates under high-frequency pulsed irradiation. The mesoscale structure of the composite also determines whether or not percolation effects occur. The quantum properties of the dot, and

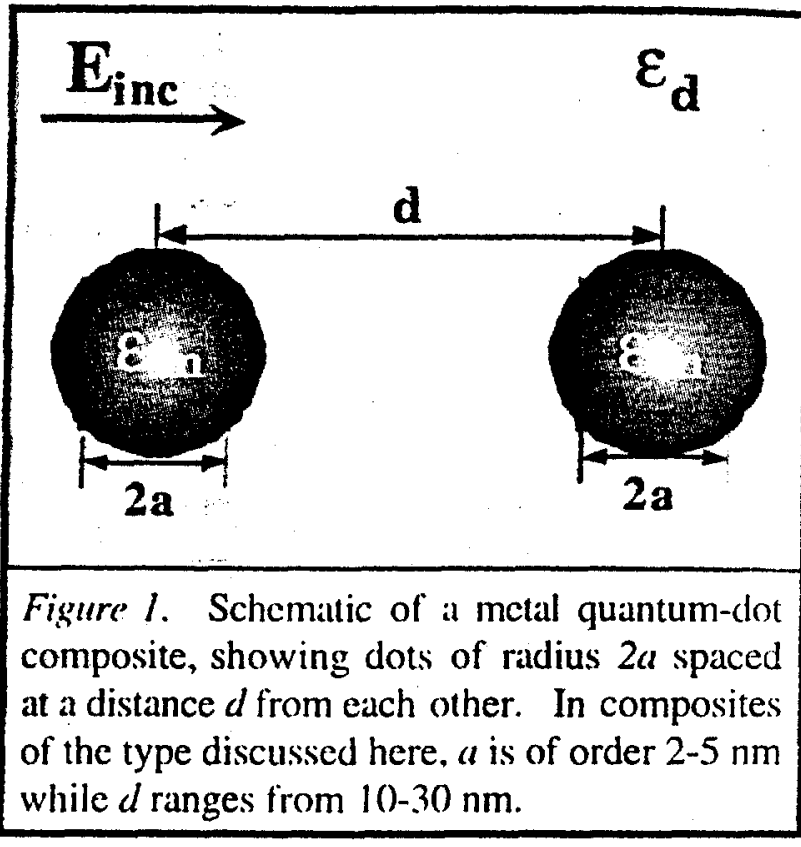
classical field effects due to the embedding of the quantum dot in an appropriate matrix, both contribute to the overall nonlinear optical response and can be optimized by matcrials processing.

Quantum-confinement effects come into play for metal quantum dots smaller than the mean free path of bulk conduction-band electrons, typically below dot diameters less than 10-13 $n m .6$ The energy spectrum for an electron in a spherical box, with bandgap energy $E_{\text {sap, }}$, principal and angular momentum quantum numbers $(n, \ell)$, momentum $(k)$ and a band index $b$ are

$$
E_{b n \ell}=\frac{E_{g a p}}{2} \pm \frac{\hbar^{2} k_{n \ell}^{2}}{2 m_{b}}
$$

In semiconductors, the differing band effective masses and the size dependence of the gap energy produce the well-known sensitivity of the optical response to size inhomogeneities. For metals. on the other hand, the band gap vanishes, and the effective masses of electrons and holes are roughly equal except for strongly localized electrons, such as the $d$-electrons. The energy-level spacing is roughly equal to the Fermi energy divided by the number of conduction-band electrons.

The relaxation time of metal quantum dot composites, measured with ultrafast lasers, is on the order of a few picoseconds, ${ }^{8}$ even near the surface plasmon resonance," with a relatively weak dependence on laser pulse energy. ${ }^{10}$ The chief mechanisms for relaxing the excitation include electron-electron scattering ( $\sim 10 \mathrm{fs})$; electron dephasing by collisions with the dot walls ( $\sim 50-80 \mathrm{fs}$. depending on quantum-dot radius), ${ }^{1}$ electron-phonon coupling in the metal dot ( 1-3 ps) and electron-phonon coupling with the surrounding dielectric ( $-100-200 \mathrm{ps})$.

The optical response of a metal quantum-dot composite is characterized by the dielectric polarization (dipole moment per unit volume). The $i$ th component of the polarization $\mathbf{P}$ induced by an applied optical field $E=E_{o} \cdot \exp [i \omega t]$ can be expanded up to third order in a power series as

$$
P_{i}=\sum_{j} \chi_{i j}^{(1)} E_{i}+\sum_{j, k} \chi_{i j k}^{(2)} E_{j} E_{k}+\sum_{j, k, l} \chi_{i j k l}^{(3)} \quad E_{j} E_{k}^{*} E_{l}
$$


where the summation indices refer to Cartesian coordinates in the material as well as the polarization of the applied optical field. In isotropic materials, the second-order term vanishes.

Classical confinement effects, in a mean-field theory, result from the oscillations of the conduction-band electrons against the ionic background charge and the restoring force of the surface, thereby altering the field in the vicinity of the dot. For a MQDC with metal dots having a complex dielectric constant $\varepsilon_{q d}(\omega)=\varepsilon_{I}(\omega)+i \varepsilon_{2}(\omega)$ occupying a volume fraction $p<<1$, and a host medium with a real dielectric constant $\varepsilon_{h}$, the first-order susceptibility $\chi^{(1)}$ is given by

$$
\chi_{\text {eff }}^{(1)}=p \cdot \chi_{q d}^{(1)} \cdot\left|\frac{3 \varepsilon_{h}}{\left(\varepsilon_{1}+2 \varepsilon_{h}\right)^{2}+\varepsilon_{2}^{2}}\right|^{2} \equiv p \cdot \chi_{q d}^{(1)}\left|f_{c}\right|^{2}
$$

where the quantity $f_{c}$ measures the local field enhancement due to the dielectric polarization. This expression has a resonance at the frequency $\omega_{r}$ for which $\varepsilon_{1}\left(\omega_{r}\right)+2 \varepsilon_{t}\left(\omega_{r}\right)=0$. This surface plasmon resonance can be shifted substantially by altering the shape and size of the nanocrystals, or by changing the combination of metal and dielectric, or by introducing a core-shell structure.

The classical confinement effect on the third-order susceptibility can be derived by applying Maxwell's equations to the nonlinear susceptibility to first order in the electric field. ${ }^{12}$ If the nanoparticle radius $a$ satisfies the inequality $\left(\omega n_{t} a / c\right)<\delta$, where $\delta$ is the skin-depth for the metal, and if $a \ll \delta$, the field is essentially constant over the entire quantum dot. Under these circumstances, the nonlinear optical susceptibility $\chi^{(3)}$ is ${ }^{13}$

$$
\chi_{c f f}^{(3)}=p \cdot \chi_{q d}^{(3)} \cdot\left|\frac{3 \varepsilon_{h}}{\left(\varepsilon_{1}+2 \varepsilon_{h}\right)^{2}+\varepsilon_{2}^{2}}\right|^{2} \cdot\left(\frac{3 \varepsilon_{h}}{\left(\varepsilon_{1}+2 \varepsilon_{h}\right)^{2}+\varepsilon_{2}^{2}}\right)^{2} \equiv p \cdot \chi_{i d d}^{(3)}\left|f_{c}\right|^{2} \cdot\left(f_{c}\right)^{2}
$$

where $f_{c}$ again measures the local field enhancement due to the polarization. A self-consistent treatment using a jellium model for the metal ${ }^{14}$ yields the same result for this special case.

The design of nonlinear optical materials and structures is constrained by the fact that the optical susceptibilities $\chi_{q d}^{(1)}$ and $\chi_{y d}^{(3)}$ of the metal quantum dot behave in opposite ways. The former increase, while the latter decrease, with quantum-dot radius. Hence, it is generally desirable (o) keep quantum-dot volume small. Also, whereas the amplitude of the plasimon resonance dac to dielectric confinement effect is proportional to the square of the field-enhancement factor for the first-order susceptibility, the third-order susceptibility is proportional to the fourth power of $f$.

Working optical devices may be based either on dispersion or absorption. The first-order susceptibility is related to the linear refractive index $n_{o}$ and absorption coefficient $\alpha_{o}$, by:

$$
n_{o}=\Re e\left[1+\chi^{(l)}\right] \quad \alpha_{o}=\frac{\omega}{n_{c} c} \operatorname{Sm}\left|\chi^{(1)}\right|
$$

For a material without a preferred symmetry axis, the composite third-order susceptibility has an analogous relationship to the nonlinear refractive index and nonlinear absorption coefficient: ${ }^{15}$

$$
n_{2}=\frac{I 2 \pi}{n_{0}} \operatorname{Re}\left[\chi^{(3)}\right] \quad \beta=\frac{96 \pi^{2} \omega}{n_{o}^{2} c^{2}} \operatorname{Im}\left[\chi^{(3)}\right]
$$

These third-order nonlinear optical properties, and their dependence on the micro- and mesoscale materials properties of the composite, are the focus of the remainder of the paper. 


\section{Figures of Merit for $\chi(3)$ Devices}

Virtually every linear electro-optic coupling, modulation or switching device can be reconfigured for all-optical operation by inserting appropriate nonlinear optical materials in waveguide or layered geometries. ${ }^{16}$ Schematic representations of some possible nonlinear all-optical switching devices are shown in Figure 2. Quantum-dot composites appear to fit neatly into the fabrication of all of these device geometries, though the application is particularly obvious in the Mach-Zehnder interferometers, for example, where one simply has to incorporate the nonlinear material in one arm of the interferometer.

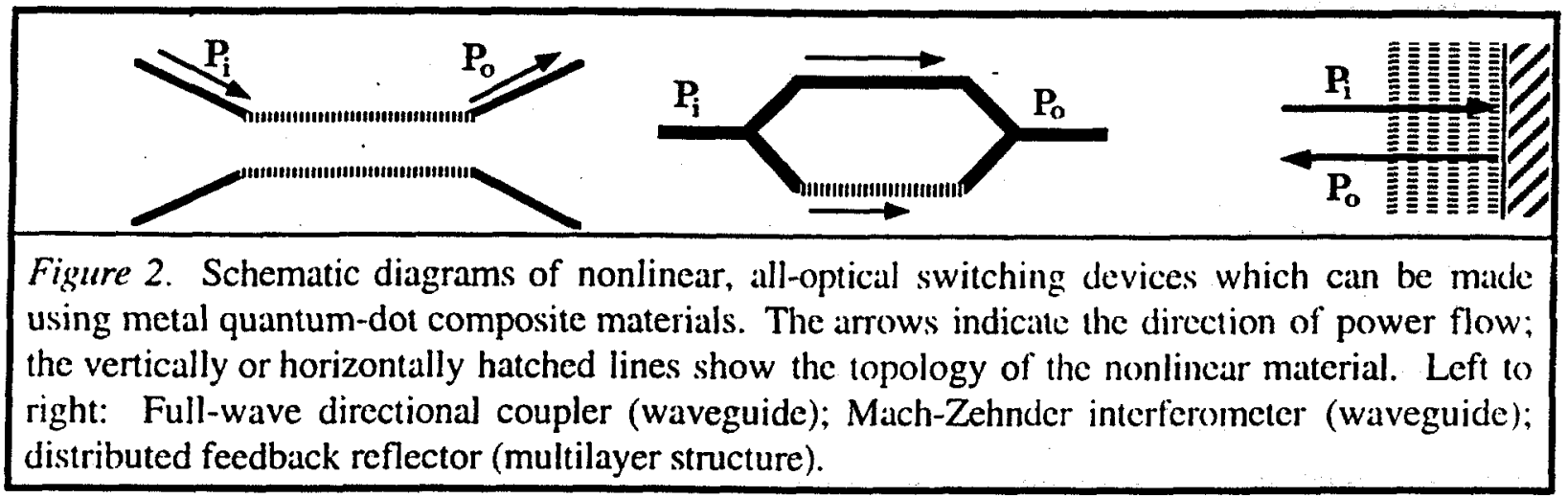

The constraints imposed on mesoscale optical properties by photonic-device structure and functionality can be expressed in a convenient, albeit approximate, way by figures of merit (FOMs). Optical figures of merit relate the magnitude of the third-order nonlinearity to the linear response and nonlinear optical response. For purely dispersive or absorptive nonlinearities, the optical figures of merit (not dimensionless!) off- or on-resonance, respectively, are: ${ }^{17}$

$$
f_{\text {disp }}=\frac{\omega \cdot n_{2}}{n_{o}} \quad f_{\text {abs }}=\frac{\beta}{\alpha_{o} \cdot \tau}
$$

The relaxation time $\tau_{\text {mat }}$ in the expression for $f_{a b s}$ is the limiting energy relaxation time of the resonant (long) or non-resonant (short) excitation process. These two quantities measure, respectively, the change in refractive index or absorption per unit time per unit intensity, thus permitting comparison of materials with widely differing excitation and relaxation mechanisms.

A defect-related dimensionless materials figure of merit arises from limits imposed by twophoton absorption (TPA) on all-optical switching; it is defined by ${ }^{18}$

$$
f_{T P A}=\frac{2 \lambda \cdot \beta}{n_{2}}<1
$$

and expresses the capacity of the nonlinear material to induce a phase shift without dropping below the critical operating intensity of the device. Other optical properties of the nonlinear composites besides TPA, ${ }^{19}$ such as the presence of optically active defects, ${ }^{20}$ may force the systems designer to accept smaller third-order susceptibilities in order to optimize overall system performance.

Functional figures of merit relate device properties, such as phase shifts, to the mesoscale materials properties. For example, in a nonlinear directional coupler or Mach-Zehnder interferometer, switching occurs if $w \equiv \Delta \beta_{\text {sar }} L / \lambda>2$, where $L$ is the interaction length, $\lambda$ is the wavelength, and $\Delta \beta_{\text {sat }}$ is the change in the guided-wave index at saturation,which usually equals the 
saturation in the material index of refraction, $\Delta n_{\text {sat }}$. However, high throughput also requires that $L \alpha<1$, where $\alpha$ is the linear absorption coefficient. Combining these two conditions yields a dimensionless figure of merit for this particular device: ${ }^{21}$

$$
W=\frac{\Delta \beta_{\text {sat }} \cdot L}{\lambda} \times \frac{1}{L \alpha}=\frac{\Delta n_{\text {sat }}}{\lambda \alpha}
$$

Note that this particular figure of merit not only includes a referent to the device type, but also its operating point, through the inclusion of the saturated value of the change in refractive index.

The operational figure of merit is effectively the number of switching operations which can be performed in one thermal relaxation time. In all-optical switching schemes, one must have a phase change of $\pi$ over some reasonable length (say, $0.1-1 \mathrm{~cm}$ for waveguide integrated-optical devices). For a device operating on nonlinear dispersion, this phase change must satisfy $\phi=2 \pi n_{2} I_{0} L / \lambda$. On the other hand, the thermal loading produced by absorption of a single pulse of duration $\tau_{\text {laser }}$ at the intensity is $\Delta Q=\alpha L I_{o} a \tau_{\text {laser }}$, where $\alpha$ is the absorption coefficient and $V=a L$ is the volume of the waveguide in which the pulse is absorbed. The figure-of-merit defining the performance of a given material at high pulse-repetition frequencies is then: ${ }^{22}$

$$
f_{\text {thermal }}=\frac{\text { Index change required for switching }}{\text { Thermal index change }}=\frac{n_{2} C_{1} \rho}{\alpha \tau(d n / d T)}
$$

where $C_{p}$ is the heat capacity at constant pressure, $\rho$ is the density, $\tau$ is the slower of the thermal and electronic relaxation times, and $d n / d T$ is the thermo-optic coefficient. Since typical thermooptic coefficients tend to fall in the range $10^{-4} \mathrm{~K}^{-1}$, the thermal refractive index change, which relaxes only very slowly in many insulators, may mask the fast electronic relaxation processes associated with $n_{2}$ in the worst cases.

A comparison of semiconductor and metal quantum-dot composites in glasss with a fuscel silica fiber shows that, by this figure-of-merit, optical libers appear to be the "best" nonlinear material. However, using several meters of optical fiber may not always be compatible with many desirable device applications! Metal QDCs and semiconductor QDCs in glasses appear to have comparable figures of merit in many cases. as shown in Table 1.

Table 1: Figures of merit for various nonlinear materials

\begin{tabular}{|c|c|c|c|c|c|}
\hline Material & $f_{\text {disv }}\left(\frac{\mathrm{w}}{\mathrm{cm}^{2} \cdot \mathrm{s}}\right)$ & $f_{a b \cdot\left(\frac{\mathrm{W}}{\mathrm{cm}^{2} \cdot}\right)}$ & $f_{T P A}$ & $\mathrm{~W}$ & $f_{\text {thurmal }}$ \\
\hline GaAs MQW & $10^{7}$ & $3 \cdot 10^{-4}$ & 6 & $\sim 2.5$ & $3 \cdot 10^{2}$ \\
\hline CdSe:glass & $10^{3}$ & 66 & 30 & 0.3 & $7 \cdot 10^{4}$ \\
\hline Cu:glass & 10 & 50 & 5 & $0.2-2.0$ & $6 \cdot 10^{4}$ \\
\hline Silica fiber & $10^{-5}$ & $10^{x}$ & 9 & $>10^{i}$ & $4 \cdot 10^{7}$ \\
\hline
\end{tabular}




\section{Experimental Details}

The drive to generate functional nonlinear optical materials is the classic synthesis vs materials characteristics problem. We shall compare three different processes which are currently in use in many laboratories to synthesize quantum-dot composites. We have omitted sol-gel processing, ion-beam-assisted deposition and classical sputter deposition processes for reasons of space.

\section{Synthesis by ion implantation}

Ion implantation can affect nonlinear device performance either by changing the optical properties of the matrix (index of refraction, for example) or the electronic structure of the metal quantum dot. A large variety of nanocomposites incorporating elemental and compound metal and semiconductor nanocrystals have been synthesized by ion implantation. Using energies ranging from $100 \mathrm{keV}$ to a few $\mathrm{MeV}$, nanocomposite layers up to a few hundred nm thick and as much as half a micron deep have been prepared. However, the process of quantum-dot nucleation and growth is poorly understood in these materials, although there is some evidence that the dots are nucleated at defect sites and grow by thermal diffusion of the implanted ions, driven by the thermal energy supplied by ion deposition. The only successful control of size distribution demonstrated so far has been achieved at the expense of deposition speed, by reducing the ion-beam current density. At low implantation current densities,

\section{Synthesis by ion exchange and ion implantation}

Planar waveguides of order $5 \mu \mathrm{m}$ thick can also be generated in one surface of a sodium silicate (soda-lime) glass by immersing the glass in a bath of molten $\mathrm{AgNO}_{3}$ or $\mathrm{CuNO}_{3}$ salts held at the eutectic point (600-700 C, depending on the salt), initiating the exchange of $\mathrm{Na}^{+}$for $\mathrm{Ag}^{+}$or $\mathrm{Cu}^{+}$, and leading to the formation of a layer of $\mathrm{Ag}$ or $\mathrm{Cu}$ ions. After ion exchange, He ions at 1.8 $\mathrm{MeV}$ energy (range $6.5 \mu \mathrm{m}$ ) at doses up to $1 \cdot 10^{16} \mathrm{~cm}^{-2}$ were implanted; the energy deposited locally by the light ions in transit is sufficient to initiate nucleation of the metal nanocrystals. Optical absorption spectra taken before and after implantation showed that the surface plasmon resonance for silver formed after the implantation, indicating that the deposition of energy by the He ions was sufficient to initiate aggregation of the dispersed $\mathrm{Ag}$ or $\mathrm{Cu}$ ions. Optical absorption spectra after implantation show a strong peak at wavelengths corresponding to the surface plasmon resonance.

\section{Synthesis by pulsed laser deposition}

The most recent developments in MQDC synthesis have been using pulsed laser deposition with multiple targets. In one recently published paper, ${ }^{5} \mathrm{Cu}$ nanocrystallites $4 \pm 1 \mathrm{~nm}$ in diameter were generated in a ten-period structure with $\mathrm{Al}_{2} \mathrm{O}_{3}$ as a matrix, by sequential laser ablation of a $\mathrm{Cu}$ metal target and a pressed-powder $\mathrm{Al}_{2} \mathrm{O}_{3}$ target. The laser used was an $\mathrm{ArF}$ laser, operating at a wavelength of $193 \mathrm{~nm}$; typical fluences were of order $2 \mathrm{~J} \cdot \mathrm{cm}^{-2}$. Particularly surprising was the fact that the spacing of the nanocrystals in each layer was highly uniform, measuring from the transmission electron microscopy $8 \pm 1 \mathrm{~nm}$. An analysis of the ablation plume from the $\mathrm{Cu}$ target showed the presence of $\mathrm{Cu}_{6}$ and $\mathrm{Cu}_{7}$ clusters, as measured by matrix-assisted laser desorptionionization mass spectrometry. Assuming that the clusters indeed arrive at the surface in this configuration, it would appear that cluster diffusion on the surface, perhaps nucleating at defect sites in the deposited alumina film (which is polycrystalline), leads to growth limited only by the surface temperature. Recent unpublished results in our laboratory have shown the presence of Cu nanoclusters in complex ternary oxides as well. 
The relative merits of these three synthesis techniques are compared in Table 2, where we have noted the possible effects of each process on the critical mesoscale properties of MQDCs. Not all of these capabilities have been fully demonstrated in every synthetic technique.

Table 2. Comparison of mesoscale materials characteristics for various beam syntheses.

\begin{tabular}{|l||c|c|c|}
\hline Mesoscale properties & Ion Implantation & $\begin{array}{c}\text { Ion Exchange } \\
\text { and Implantation }\end{array}$ & $\begin{array}{c}\text { Pulsed Laser } \\
\text { Deposition }\end{array}$ \\
\hline \hline Control of size distribution & Marginal & Moderate & Good \\
\hline Small quantum-dot size & Marginal & Good & Excellent \\
\hline Core-shell structures & Yes & No & Possible? \\
\hline Waveguides & Too shallow & Yes & Yes \\
\hline Lateral structuring & With great difficulty & Probably not & Possible? \\
\hline Defect density & High & Low & Low \\
\hline High thermal conductivity & Sometimes & Marginal & Yes \\
\hline
\end{tabular}

\section{Nonlinear optics experiments}

Nonlinear optical measurements were carried out using a tunable. synchronously pumped. mode-locked and cavity-dumped dye laser with an average output power of $100-250 \mathrm{~mW}$ at a pulsc repetition frequency of $0.76-19 \mathrm{Mhz}$. The dye laser was pumped by a continuous-wave. modelocked and frequency-doubled Nd:YAG laser producing $100 \mathrm{ps}$ pulses at a pulse repetition frequency of $38 \mathrm{Mhz}$. The pulse duration of the dye laser output beam was measured by standard autocorrelation techniques to be $6 \mathrm{ps}$ (using Rhodamine 6G) or $0.7 \mathrm{ps}$ (using Pyluromethene 570). The focusing lens in both experiments was $15 \mathrm{~cm}$, leading 10 a peak focal-spot intensity on the order of $2 \cdot 10^{\mathrm{X}} \mathrm{W} \cdot \mathrm{cm}^{-2}$.

The third-order susceptibility of the MQDCs was measured directly by degencrate four-wave mixing in the forward, phase-matched geometry. The beam from the dye laser was split into three beams: two strong pump beams, each with approximatcly $45 \%$ of the lotal inlensity. and a single weak probe beam with the remaining laser output. The pump beams were aligned onto the salmple and precisely overlapped in space and time; the probe beam was reflected fiom a corner cube oll a movable translation stage, and was varied in time to sweep through the probe beanns as they struck the sample. The phase-conjugate signal, strong enough to be visible to the naked eye, was identified from the direction consistent with conservation of momentum. and was routed to an uncooled photomultiplier detector the output of which was fed to a dual gated photon counter.

The nonlinear index of refraction $n_{2}$ and nonlinear absorption $\beta$ of the samples were measured using a single-beam technique, the so-called $Z$-scan, in which phase distortion is converted to amplitude distortion as a well-characterized Gaussian beam is directed through a fixed. long-focallength lens to the sample, located near the focal plane. As the sample is scanned along the optical axis of the system through the focal plane, the intensity on the sample is varied. A Z-scan measurement of both near- and far-field intensities yields the nonline:ur index and nonlinear absorption from a single measurement. 
For a thin nanocluster layer embedded in a transparent dielectric and for a high-repetition-rate laser, the relative far-field transmitted power is determined by both the optical response of the metal particle and the surrounding dielectric, and by the two-dimensional thermal relaxation processes through which the absorbed laser energy flows out of the illuminated region. In the limit of small nonlinearities, the power transmitted to the detector is given by

$$
P_{d e t}=\left[\frac{1}{1+\beta l_{o} L \cdot \frac{1-\exp (-\alpha L)}{\alpha}}\right] \cdot\left[1-\frac{2 C_{1 \zeta}}{\left(1+\zeta^{2}\right)^{2}}+\frac{2 C_{2} \zeta}{\left(1+\zeta^{2}\right)}\right]
$$

where $C_{1}$ and $C_{2}$ are propertional to the electronic and thermal components of the nonlinear index, respectively, and $\zeta$ is a normalized $z$-coordinate along the optical axis. The differing zdependences of the electronic and thermal terms in Eq. (11) arise because the electronic component of the nonlinear index is proportional to intensity, while the thermal nonlinear index is proportional to the temperature rise and hence to the total power.

\section{Mesoscale Engineering Issues}

We have seen that the nonlinear performance of MQDCs depends in a complicated way on the optical properties of the quantum dots and the matrix - as in the mean-field enhancement factor $f_{r}$. But the figures of merit also include the material relaxation factors and the thermal properties of the composite. Engineering the materials for even the simplest devices requires an understanding of which parameters control which property or group of properties. In this section, we examine several different synthesis variables and their effects on nonlincar optical performance.

In trying to produce third-order nonlinear optical materials for practical applications, there are several interrelated characteristics of the MQDCs, each imposing unique constraints on the quantum dots and the matrix in which they are embedded. These issues may be summarized as follows:

- Spectral and temporal response of the metal quantum dot, controlled chiefly by composition and size;

- Spectrum and amplitude of the surface plasmon resonance, controlled by the physical structure of the quantum dot and the dielectric functions of metal and matrix;

- Thermal relaxation time of the nanocomposite, governed primarily by the thermal transport properties of the matrix and the size of the quantum dot.

As will be demonstrated in the succeeding paragraphs, the constraints imposed by mesoscale materials properties can be altered in a number of interesting ways using energetic beams to control the microscopic (i.e., atomic scale) properties of the quantum dot, interface and matrix.

\section{Quantum-dot optical response}

Semiconductor quantum dots achieve wavelength tunability by varying the quantum-dot size: As the size of the dots is progressively reduced below the Bohr exciton radius, the bandgap energy shifts progressively toward the blue; moreover, the electron and hole effective masses are also size dependent. While the oscillator strength is large for any specific optical transition, the bands are nearly parabolic, meaning that there is relatively little anharmonicity, and the increase in transition probability comes almost exclusively from enhanced oscillator strength. In metal quantum dots, on 
the other hand, tunability is low, because energy levels are spaced almost equidistant. The anharmonicity is high, on the other hand, because the confining potential is very deep and nearly a square well. The only resonant enhancement is provided by either quantum confinement effects on the intraband transition, or by the surface plasmon resonance.

The spectral and temporal response of metal quantum dots is significantly affected by whether the optical transition is an intraband or interband transition. The holes created in interband transitions are, for noble metals, in the $d$ band and are effectively screened by the $s-p$ conduction and electrons. Also, because the $d$ orbitals are already relatively strongly localized, the electronic structure of the quantum dots controls their frequency response as well as the relative size of the third-order susceptibility.

We measured the intrinsic response time of quantum dots excited via the intraband transition in $\mathrm{Au}: \mathrm{SiO}_{2}$, and compared it to the response time on the interband transition for $\mathrm{Cu}: \mathrm{SiO}_{2}$, both at a laser wavelength of 575 $\mathrm{nm}$. The experiment was a four-wave mixing measurement, in which the phase-conjugate signal for the two pump and probe beams produces a coherent signal proportional to the relaxation time of the grating created by the pump beams. The measured autocorrelation width of the laser pulse was $730 \mathrm{fs}$. The phase-conjugate signals show distinct differences in the two cases: On the interband transition for $\mathrm{Cu}$ quantum dots, the dephasing signal is symmetrical with respect to zero time

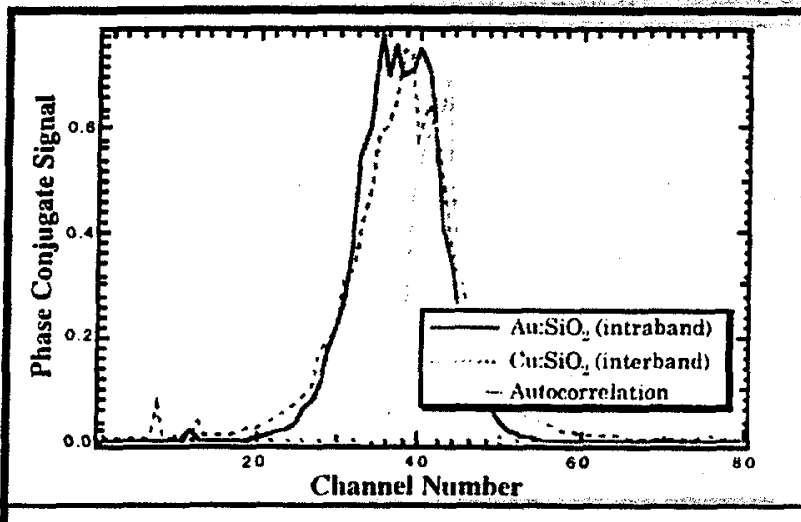

Figure 3. Degenerate four-wave-mixing signal for $\mathrm{Au}: \mathrm{SiO}_{2}$ and $\mathrm{Cu}: \mathrm{SiO}_{2}$ MQDCs, showing the finite relaxation time for the nanocrystals compared to the laser pulse ( $730 \mathrm{fs}$ ). delay (channel 40), indicating a constant relaxation rate probably due to electron-phonon coupling: the measured relaxation time is approximately $2.2 \mathrm{ps}$. In the case of the $\mathrm{Au}: \mathrm{SiO}_{2}$ sample, the phase-conjugate signal shows a pronounced asymmetry, and a flat top with a width of $1.2 \mathrm{ps}$. This is probably due to Pauli blocking: electrons excited out of conduction-band levels near the Fermi surface have fewer states available into which they can scatter - a consecpuence of the small size of the nanocrystal. This causes the electron-phonon relaxation rate to slow down as the encrgies of the scatter electrons drop down closer to the Fermi level. Both of these times are much shorter than the thermal relaxation time, so that it is the latter which is the rate-limiting process in the high-frequency response of the quantum dots.

\section{Surface plasmon resonance}

The increased nonlinear response made possible by the surface plasmon resonance (SPR) is it primary advantage of metal quantum dots for nonlinear optical applications. As shown by Eq. (3). the enhancement $f_{r}$ in the optical response from the surface plasmon is governed by the relationship between the dielectric functions of the metal and the host dielectric. Changing that relationship alters the strength and frequency characteristics of the resonance, as shown in Figure 4. Here $\mathrm{Cu}$ and $\mathrm{Ag}$ ions were coimplanted in a fused-silica matrix, with beam energies adjusted to insure that the implantation depth was equal for both species. The driving force for the alloying is believed to be the heat deposited in the matrix by the second implantation. Alloy nanoclusters were formed. and the resulting dielectric functions differed in each case. As Fig. 4 shows, the strength of the plasmon resonance near $410 \mathrm{~nm}$ (the position of the SPR for pure Ag nanocrystallites) was clearly 


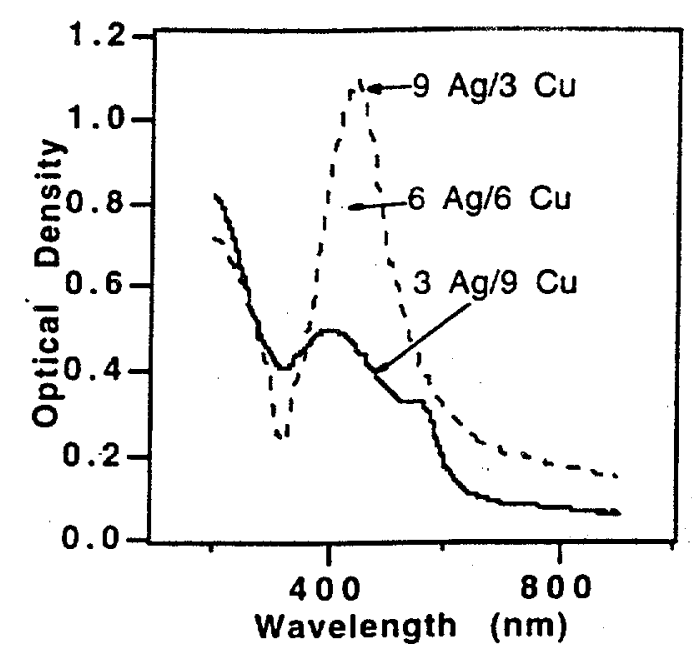

Figure 4. Optical absorption spectrum of an $\mathrm{Ag} / \mathrm{Cu}: \mathrm{SiO}_{2}$ nanocomposite, showing the effect of the increasing fraction of $\mathrm{Cu}$ relative to $\mathrm{Ag}$ on the plasmon resonance. altered by the co-implantation. Other strategies which can potentially increase the strength of the plasmon resonance include embedding the nanocrystals in a nonlinear dielectric, because the intrinsic dipole moment then adds to the local field enhancement produced by the plasmon resonance. Interestingly, the nonlinear response was also altered, with modest decreases in the nonlinear index but with a significant change in the nonlinear absorption. Such a change. of course, will alter some of the figures of merit governing the performance of the material.

The central fiequency and strength, as well as the width, of the plasmon resonance can also be altered by creating core-shell nanostructures. In this calse, a proper match of the boundary conditions across the corc, shell and matrix interface can produce an even langer field enhancement than is possible for a single-element nanocrystal in a dielectric. This effect is shown in Figure 5 , in which Ag was co-implanted with several different metals to produce core-shell structures in the two cases (Sh) and Cd) which are not miscible with Ag.

\section{Thermal Transport Properties}

The performance figure-of-merit introduced carlier probably poses the greatest single matterials challenge to the construction of practical third-order metal-quantum-dol composites. At high frequencies, the residual thermal loading can be substantial, even al relatively low powers: the problem is compounded by the fact that the dielectric incdium in which the quantum dots are embedded is typically a poor conductor of heat. Previous studies of the nonlinear index of refraction showed that the thermal component of Eq. (18) comes to dominate the electronic component at relatively low pulse repetition frequencies. For example, Figure 6 shows how the near-field and far-field Z-scans vary with pulse repetition frequency for $\mathrm{Au}$ nanocrystallites in fused silica, synthesized by ion implantation at a total ion dose of approximately $10^{17} \mathrm{Au}$ ions $\cdot \mathrm{cm}^{-2}$. At a pulse repetition frequency above $10 \mathrm{MHz}$, the dominance of the thermal component is shown by the sudden rise in the peak-to-valley ratio of the farfield Z-scan [Figure 6(a)] and by the shift from nonlinear absorption to nonlinear saturation [Figure $6(\mathrm{~b})]$ in the near-field Z-scan.

This problem can be overcome by embedding the quantum dots in a medium with higher thermal conductivity than is typical for glasses.

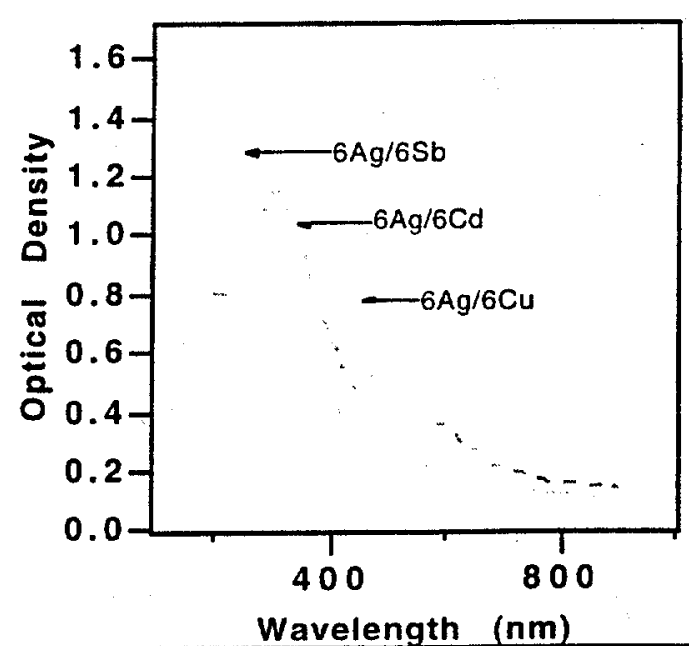

Figure 5. Optical density $v s$ wavelength for a sample implanted with two differing ion species showing the shifting of the surface plasmon resonance with composition. 


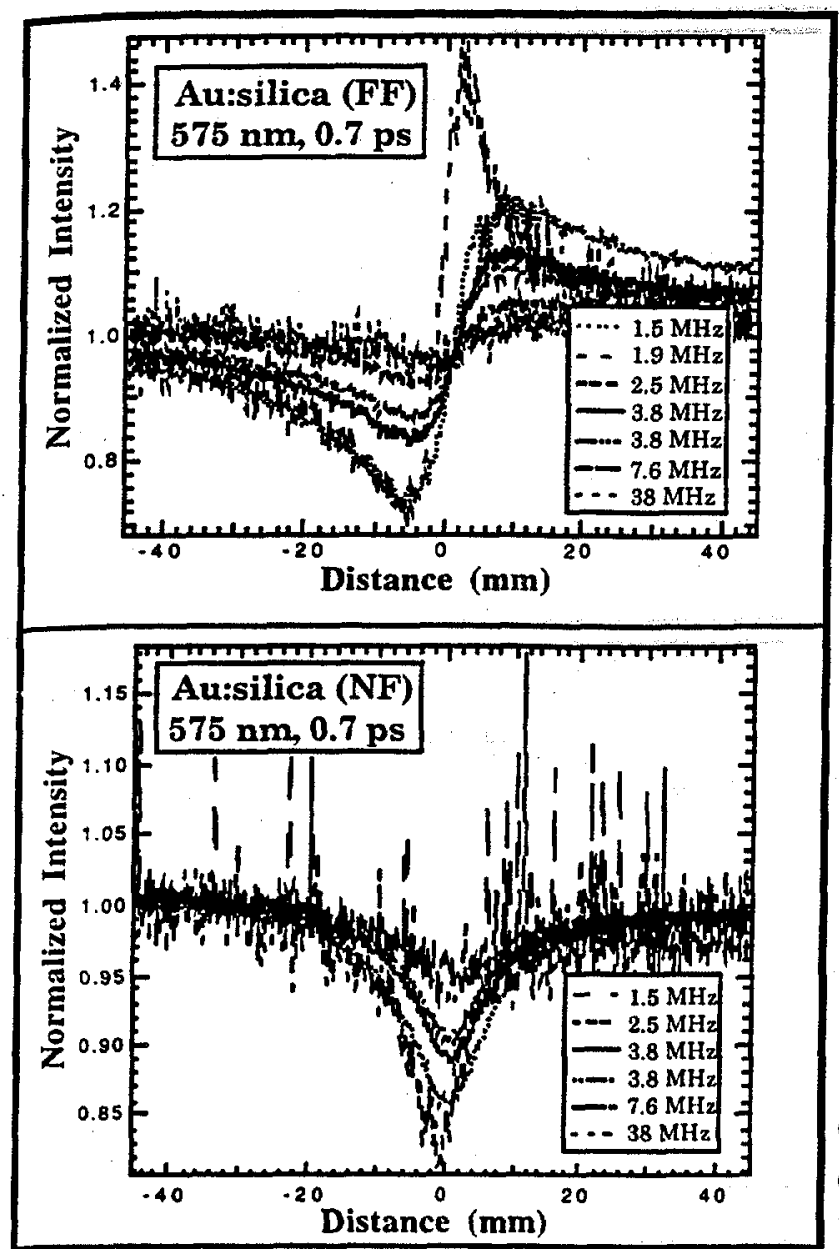

Figure 6. Z-scans showing far- and near-field transmission (upper and lower panels, respectively) of an $\mathrm{Au}: \mathrm{SiO}_{2} \mathrm{MQDC}$, as a function of pulse repetition frequency.

In another experiment, $\mathrm{Cu}$ quantum dots were embedded in $\mathrm{Al}_{2} \mathrm{O}_{c}$ by pulsed laser deposition, as described above, and the near-field and far-field scans were used to determine the relative size of thermal and electronic effects on the nonlinear index and absorption. These data were then compared with earlier published data on the relative sizes of the thermal and electronic components of the nonlinear index, and with the nonlinear absorption. The $\mathrm{Cu}: \mathrm{Al}_{2} \mathrm{O}_{3}$ composite was found to have a somewhat lower thermal to electronic component than the $\mathrm{Cu}: \mathrm{SiO}_{2}$ composite. However, the improvement in the nonlinear absorption was much more dramatic: the saturable absorption decreased by two orders of magnitude in the $\mathrm{Cu}: \mathrm{Al}_{2} \mathrm{O}_{3}$ material! This suggests that embedding the nanocrystals in even higherconductivity matrix materials should lead to significant improvements in the high-pulserepetition-frequency performance of these nanocomposites. We have recently begun experiments aimed at doing just that, by using pulsedlaser-deposition synthesis to nucleate and yrow $\mathrm{Cu}$ quantum dots in indium-tin oxide, a transparent, electrically conductive oxide with a conductivity over two orders of magnitude larger than that of alumina.

In recent computational studies of the thermal conductivity of nanocluster composites, it was found that the transport of heat across the boundary cannot be adequately described by standard Fourier heat-conduction theory once the size of the nanocrystals drops below the meanfree path of the heat carriers. As it turns out, the thermal conductivity is reduced by as much as a factor of two over bulk values, so that as the nanocrystallites are reduced in size. the heat transport tends to be described not by continuum Fourier heat conduction theory, but by a microscopic Boltzmann transport formalism. It remains to be seen precisely what the tradeoff is between the increased nonlinear-optical performance derived from smaller cluster sizes and the penalty for highfrequency operation incurred by the reduced thermal conductivity of the smaller nanoclusters.

\section{Conclusions}

In summary, we have described a number of the microscopic and mesoscale materials properties of metal quantum-dot composites which can be influenced by cnergetic laser and ion beams. Nonlinear optical experiments show clearly that these properties are directly influenced by these atomic-scale properties. Hence, future studies of materials will need to take these properties into account if they are to produce meaningful progress toward nanocomposites suitable for photonics applications. In particular, the question of thermal transport is critical and needs to be addressed. 


\section{Acknowledgement}

Research at Vanderbilt is partially supported by the Army Research Office under contract DAAH04-93-G-0123; research at Padua is supported by the Institute of Materials Physics of the Italian National Research Council (INFM-CNR). G. Luepke is supported by a Feodor-Lynen Fellowship of the Alexander von Humboldt Foundation. The Oak Ridge National Laboratory is partially supported by the U. S. Department of Energy, Division of Basic Energy Sciences, under contract DE-AC05-96OR22464 with Lockheed-Martin Energy Systems, Inc.

\section{References}

1. C. Buchal, S. P. Withrow, C. W. White and D. B. Poker. Amm. Rev. Mat. Sci. 24 (1994) 125.

2. P. Mazzoldi, G. W. Arnold, G. Battaglin, F. Gonella and R. F. Haglund, Jr., J. Nonlincar Opt. Phys. and Matls. 5 (1996), 285.

3. G. De, L. Tapfer, M. Catalano, G. Battaglin, F. Gonella, P. Mazzoldi and R. F. Haglund, Jr., Appl. Phys. Lett. 68 (1996) 3820.

4. H. B. Liao, R. F. Xiao, J. S. Fu, P. Yu, G. K. L. Wong and P. Sheng. Appl. Phys. Lett. 70 (1997), 1-3. 1. Tanahashi, Y. Manabe, T. Tohda, S. Sasaki and A. Nakamura, J. Appl. Phys. 79 (1996) 1244.

5. J. M. Ballesteros, R. Serna, J. Solís, C. N. Alonso, A. K. Petford-Long, D. H. Osborne and R. F. Haglund, Jr., Appl. Phys. Lett. 71 (1997) 2409.

6 F. Hache, D. Ricard and C. Flytzanis, J. Opt. Soc. Am. B 3 (1986) 1047.

7. S. Schmitt-Rink, D. A. B. Miller, and D. S. Chemla, Phys. Rev. B 35 (1987) 8113.

8. T. Tokizaki, A. Nakamura, S. Kaneko, K. Uchida, S. Omi, H. Tanji and Y. Asahara, Appl. Phys. Lett. 65 (1994) 941.

9. M. Perner, P. Bost, U. Lemmer,'G. von Plessen, J. Feldmann, U. Becker, M. Mennig, M. Schmitt and H. Schmidt, Phys. Rev. Lett. 78 (1997) 2192.

10. J.-Y. Bigot, J.-C. Merle, O. Cregut and A. Daunois, Phys. Rev. Lett. 75 (1995) 4702.

11. R. F. Haglund, Jr., Li Yang, D. H. Osborne, H. Hosono, C. W. White and R. A. Zuhr, Appl. Phys. A 62 (1996) 403.

12. D. Stroud and V. E. Wood, J. Opt. Soc. Am. B 6 (1989) 778.

13. D. Stroud and P. M. Hui, Phys. Rev. B 37 (1988) 8719.

14. F. Hache, D. Ricard and C. Girard, Phys. Rev. B 38 (1988) 7990.

15. M. J. Weber, D. Milam, D. and W. L. Smith, W. L., Opt. Eng. 17 (1978) 463.

16. G. I. Stegeman, E. M. Wright, N. Finlayson, R. Zanoni. R. and C. T. Seaton, IEEE J. Lightwave Tech. 6 (1988) 953.

17. C. Flytzanis, F. Hache, M. C. Klein, D. Ricard and Ph. Roussignol, Prog. Optics 29 (1991) 323.

18. V. Mizrahi, K. W. DeLong, G. I. Stegeman, M. A. Saifi and M. J. Andrejco, Opt. Lett. 14 (1989) 1140.

19. J. W. Aitchison, M. K. Oliver, E. Kapon, E. Colas, and P. W. E. Smith, Appl. Phys. Lett. 56 (1990), 1305.

20. K. W. DeLong, V. Mizrahi, G. I. Stegeman, M. A. Saifi, and M. J. Andrejco, Appl. Phys. Lett. 56 (1990) 1394.

21. G. I. Stegeman and R. H. Stolen, J. Opt. Soc. Am. B 6 (1989) 652.

22. S. R. Friberg and P. W. Smith, IEEE J. Quantum. Electron. 23 (1987) 2089. 